\title{
Zu viel rotes Fleisch macht Frauenherzen schwach
}

\author{
Eine eiweißreiche Ernährung mit vorwiegend rotem Fleisch als Proteinquelle scheint \\ bei Frauen über 50 das Herzinsuffizienz-Risiko zu erhöhen.
}

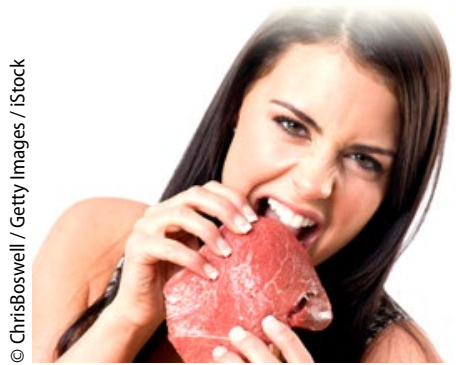

Wie lange macht ihr Herz das mit?

Wenn die Teilnehmerinnen der Women's Health Study die Diätempfehlungen der American Heart Association beachtet hätten, hätte ihre Herzkraft vielleicht weniger stark nachgelassen.
Die AHA empfiehlt nämlich Früchte, Obst, Vollkorn, Geflügel, Fisch und Nüsse, und sie rät dazu, rotes Fleisch sowie gezuckerte Speisen und Getränke nur in Maßen zu genießen.

Die Autoren der vorliegenden Studie analysierten die selbstberichteten Ernährungsgewohnheiten von fast 104.000 Teilnehmerinnen der Women's Health Study. Gleichzeitig überprüften sie anhand von Biomarkern die Angaben. Dazu maßen sie die Stickstoffausscheidung im Urin und berechneten den Energieverbrauch über die $\mathrm{CO}_{2}$-Abgabe.
1.711 Frauen entwickelten im Beobachtungszeitraum eine Herzinsuffizienz. Mithilfe der Cox-Regression berechneten die Autoren die Assoziation zwischen Proteinkonsum und Herzinsuffizienzrisiko. Ergebnis: Unabhängig von anderen Einflussgrößen erhöhten Liebhaberinnen von Kotelett, Schnitzel und Steak ihr Herzinsuffizienzrisiko substanziell. Ein erhöhter Konsum von Proteinen aus pflanzlichen Quellen schien dagegen einen gewissen Schutzeffekt auszuüben. - DE

- AHA-Kongress, New Orleans, 12.-16. November 2016

\section{GLAGOV-Studie}

\section{PCSK9-Inhibitor verkleinert Koronarplaques}

\author{
In der ersten intrakoronaren Bildgebungsstudie mit einem PCSK9-Hemmer \\ ließ der Antikörper Koronarplaques schrumpfen.
}

_ LDL-Cholesterinsenkung mit Statinen reduziert bei kardiovaskulären $\mathrm{Pa}$ tienten das Risiko für Herzinfarkt, Schlaganfall und Herztod. In Studien mit intravaskulärem Ultraschall (IVUS) zeigte sich unter Statintherapie eine Verlangsamung der Progression bzw. einer Regression koronarer Atherome.

Die jetzt verfügbaren PCSK9-Inhibitoren können LDL-Werte bei Patienten unter Statintherapie noch einmal halbieren. Dadurch sind weit tiefere LDLWerte erreichbar als bisher. Ob die zusätzliche Therapie mit diesen Antikörpern noch mehr klinische Endpunkte verhindert als Statine allein, wird die FOURIER-Studie im Frühjahr 2017 zeigen.

Jetzt ergab die beim US-Herzkongress (AHA 2016) präsentierte GLAGOV-Stu- die, dass Evolocumab (Repatha ${ }^{\circledR}$ ) den Schrumpfeffekt in den Koronarplaques verstärkt.

\section{Atherosklerose rückläufig}

In der doppelblinden Studie waren 968 Patienten mit symptomatischer koronarer Herzkrankheit und durchschnittlichen LDL-Werten von $93 \mathrm{mg} / \mathrm{dl}$ unter Statintherapie mit Evolocumab oder Placebo 18 Monate lang behandelt worden. Die Statin-Behandlung wurde fortgeführt. In der Evolocumab-Gruppe fiel das mittlere LDL um 60\% auf 36,6 mg/ $\mathrm{dl} \mathrm{ab}$, in der Kontrollgruppe blieb der LDL-Wert im Schnitt bei $90 \mathrm{mg} / \mathrm{dl}$.

Die primäre Aufmerksamkeit galt dem Endpunkt „Percent Atheroma Volume“ (PAV). Die PAV nahm in der Kontrollgruppe um $0,05 \% \mathrm{zu}$ und in der
Evolocumab-Gruppe um 0,95\% ab ( $\mathrm{p}<$ 0,0001), berichtete Studienautor Prof. Steven Nissen von der Cleveland Clinic.

\section{LDL-Spiegel von $24 \mathrm{mg} / \mathrm{dl}$ in Subgruppe}

Nissen analysierte Patienten, die eingangs schon LDL-Werte unter $70 \mathrm{mg} / \mathrm{dl}$ aufwiesen, separat. Bei diesen Patienten erreichte die Evolocumab-Therapie ein LDL von im Schnitt 24 (!) mg/dl. Patienten mit solch extrem niedrigen LDLWerten zeigten unter dem PCSK9-Hemmer eine PAV-Abnahme von 1,97\% . Dies ging bei $81 \%$ der Patienten mit einer Regression der Plaques einher. Die Behandlung wurde gut vertragen.

Dr. med. Dirk Einecke

- AHA-Kongress, New Orleans, 12.-16. November 2016 Nicholls SJ et al. JAMA 2016, doi: 10.1001/jama.2016.16951 